\title{
Precise Evaluation of Coaxial to Waveguide Transitions by Means of Inverse Techniques
}

\author{
Antonio José Lozano-Guerrero, Francisco Javier Clemente-Fernández, Juan Monzó-Cabrera, Member, IEEE, \\ Juan Luis Pedreño-Molina, and Alejandro Díaz-Morcillo, Senior Member, IEEE
}

\begin{abstract}
In this paper, a new two-tier inverse characterization technique for coaxial to waveguide transition evaluation is presented and properly validated. The transition is characterized by estimating its scattering parameters and a cascade procedure is employed in order to compare simulations and measurements during the unterminating procedure. In contrast to other unterminating methods there are no restrictions concerning the number and type of standards and two transitions are simultaneously characterized. Additionally, genetic algorithms and the gradient descent method are used for error minimization during the unterminating stage. The accuracy of this two-tier inverse technique is evaluated as a function of the employed standards and the obtained results are compared to those provided by different well-known calibration algorithms. Results show that it is possible to properly characterize the coaxial to waveguide transition in a very flexible and accurate way.
\end{abstract}

Index Terms-Coaxial to waveguide transitions, genetic algorithms, inverse measurement, two-tier calibration.

\section{INTRODUCTION}

$\mathbf{T}$ RANSITIONS are a key part of most microwave systems and circuits, making possible the interconnection between different types of transmission lines such as waveguides, coaxial, coplanar, or microstrip lines. Hence, it is imperative to have a good characterization of these structures in order to assess their performance and to provide information that can be used during the design, measurement, or optimization processes. Therefore, a large number of contributions can be found in the technical literature regarding the modeling and characterization of various types of transitions, especially coaxial to microstrip [1]-[10] and coaxial to waveguide transitions [11]-[14]. Several methods of adapter characterization can be found in [15] and [16] paying special attention to the study of uncertainties.

In particular, precise evaluation of coaxial to microstrip transitions is mandatory to obtain accurate measurements of microstrip integrated circuits by means of a vector network analyzer (VNA) since the quality of the measurements is strongly related to the quality of the transitions and repeatability is a critical issue [9]. In fact, many times it is not possible to directly

Manuscript received April 27, 2009; revised October 15, 2009. First published December 18, 2009; current version published January 13, 2010. This work was supported in part by the Ministerio de Ciencia e Innovación under Project CIT-020000-2008-43.

The authors are with the Departamento de Tecnologías de la Información y las Comunicaciones, Universidad Politécnica de Cartagena, Cartagena E-30202, Spain (e-mail: Antonio.lozano@upct.es; Francisco.clemente@upct.es; juan.monzo@upct.es; Juan.PMolina@upct.es; alejandro.diaz@upct.es).

Digital Object Identifier 10.1109/TMTT.2009.2036408 measure the scattering parameters of a circuit implemented in microstrip or waveguide technology since VNAs work with coaxial connectors and, therefore, their measurements always include the contributions of the necessary in-between transitions.

For instance, test fixtures use coaxial to microstrip transitions in order to measure embedded microstrip circuits. If the scattering parameters of test fixture transitions are known, they can be removed from VNA measurements by the so-called deembedding process [17], [18]. However, the measurement of transition parameters is not straightforward and they must be characterized from measurements made at the VNA reference plane when known standards are embedded in the fixture. This process is referred to as unterminating [18].

There are many possibilities for unterminating depending on the standards types and error minimization procedures. For instance open-short-load (OSL) or thru-reflect-line (TRL) standards may be used [17], [18] for unterminating. However, several studies based on iterative approaches [18], [19] show that it is possible to use redundant standards in order to increase accuracy versus conventional calibration procedures.

Very important efforts have been made at the National Institute of Standards and Technology (NIST), Boulder, CO, in order to develop software packages for calibration and unterminating processes. For instance, MultiCal and StatistiCAL ${ }^{1}$ freeware software packages implement calibration algorithms based on several studies such as [17]-[22] that perform both one- and two-tier deembedding. The two-tier calibration procedure can be used to electrically characterize probe heads or other components such as coaxial to waveguide transitions and can handle up to 40 different standard types.

Coaxial lines to waveguide junctions have also been the subject of active research in recent years since they are used in a multitude of microwave applications. This kind of junctions can be divided into two different, though closely related, groups, namely, T-junctions and coaxial line to waveguide transitions. Waveguide T-junctions are commonly used in multiplexers and diplexers [23], [24] and power dividers [25], whereas transitions, which can also be viewed as a particular case of T-junctions, are used, for example, in the input and output ports of microwave cavity filters [26], [27] and in horn antennas [28].

All the above applications require extracting the scattering parameters of the electrical transitions. Thus, numerous methods and techniques have been developed using different

${ }^{1}$ MultiCal and StatistiCAL are available online at http://www.nist.gov/ eeel/electromagnetics/related-software.cfm. MultiCal and StatistiCAL are trademarks of the National Institute of Standards and Technology (NIST), Boulder, CO. 
approaches. Computer-aided design (CAD) models of connectors and transitions have been used both in coaxial to microstrip [6] and coaxial to waveguide transitions [25]. The main problem of this approach is that full-wave electromagnetic analysis of these structures requires complex frequency-dependent calculations, even if the complexity of the structure is not high. That is why some different characterization methods based on $S$-parameter measurements have been presented, specifically focused on coaxial probe modeling in waveguides and cavities.

The probe-excited waveguide problem has been studied during the last 50 years. One of the most important contributions is presented in [11], where a rigorous method to obtain the two-port scattering matrix of a probe-excited semi-infinite waveguide is shown. This procedure is known as the three-cavity moment method and makes use of three cavities and their input reflection coefficients for obtaining three linear equations with which the two-port scattering matrix can be calculated, by impressing the incident wave in the coaxial line only. The main drawback of this method is a restriction regarding the phases of the reflection coefficient of the short-circuited waveguide sections, which must not have $360^{\circ}$ differences at a given frequency. The authors suggest using phase differences of $120^{\circ}$ and $240^{\circ}$. Furthermore, this procedure assumes only one propagating mode and cannot compute generalized $S$-parameters so an extension is presented in [13]. In that study, the authors include the use of the orthogonal expansion method for modeling cylindrical posts in rectangular waveguides. Further research on the three-cavity approach can be found in [14], where a coaxial line to a rectangular waveguide junction is analyzed using the five-cavity moment method in combination with network cascading techniques and an interpolation method. All these methods use waveguide short-circuit standards at different electrical lengths from the transition in order to extract its behavior versus frequency. In this study, we present a new inverse characterization technique in order to evaluate coaxial to waveguide transitions. This new unterminating procedure is carried out by minimizing the error between simulations of scattering matrix concatenations that contain the transition as an unknown parameter and measurements of several structures that reproduce the simulated scenarios. In this study, two different standard types such as short circuits and lines are used and two transitions are simultaneously characterized. The accuracy of the inverse technique is evaluated as a function of the employed standards, and the obtained results are compared to those provided by different well-known calibration algorithms.

\section{InVERSE TeChNique USED FOR THE TWO-TIER CALIBRATION ALGORITHM}

An inverse technique [29] provides the estimation of an unknown parameter by comparing the experimental response of the analyzed structure to the simulation of the experimental scenario. In this study, the two-port scattering matrices $(S)$ of two different coaxial to waveguide transitions are simultaneously obtained by means of an inverse procedure. A specific study of a coaxial-waveguide transition has been carried out although the proposed procedure can be readily extended to other transition types. The experimental setup considers up to three waveguide

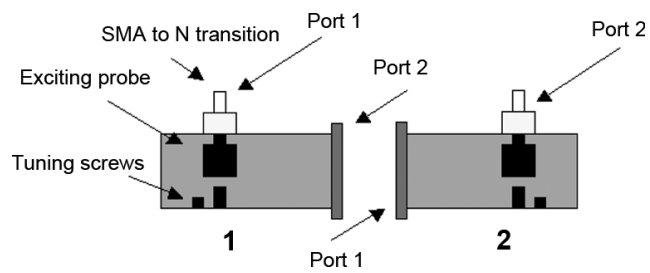

Fig. 1. Coaxial to waveguide transitions under study.

shorts and three waveguide lines with different lengths as calibrating standards placed at the end or in-between the coaxial to waveguide transitions. Therefore, this inverse procedure can be classified as a two-tier calibration problem [30]. In Fig. 1, a scheme of the WR-340 transitions under study is depicted. As can be observed, these transitions include several tuning screws in order to obtain good matching levels within the operating bandwidth.

\section{A. Parameter Description and Cascade Procedure}

The two-port scattering parameters of each coaxial to waveguide transition are modeled through their magnitude and phase representation. This leads to 12 different unknown real parameters when considering both transitions and the fact that $S_{12}^{\mathrm{tr} 1}=$ $S_{21}^{\mathrm{tr} 1}$ and $S_{12}^{\mathrm{tr} 2}=S_{21}^{\mathrm{tr} 2}$ due to reciprocity. It also must be taken into account that each scattering parameter must be evaluated at different frequency values within the studied bandwidth. The $S$ matrices for each transition are defined as follows:

$$
\begin{aligned}
& {\left[\begin{array}{ll}
S_{11}^{\operatorname{tr} 1} & S_{12}^{\operatorname{tr} 1} \\
S_{21}^{\operatorname{tr} 1} & S_{22}^{\operatorname{tr} 1}
\end{array}\right]=\left[\begin{array}{ll}
A e^{j \phi_{1}} & B e^{j \phi_{2}} \\
B e^{j \phi_{2}} & C e^{j \phi_{3}}
\end{array}\right]} \\
& {\left[\begin{array}{ll}
S_{11}^{\operatorname{tr} 2} & S_{12}^{\operatorname{tr} 2} \\
S_{21}^{\operatorname{tr} 2} & S_{22}^{\mathrm{tr} 2}
\end{array}\right]=\left[\begin{array}{ll}
D e^{j \phi_{4}} & E e^{j \phi_{5}} \\
E e^{j \phi_{5}} & F e^{j \phi_{6}}
\end{array}\right]}
\end{aligned}
$$

where $\operatorname{tr} 1$ and $\operatorname{tr} 2$ refer to each transition, $A, B, C, D, E, F \in$ $[0,1]$ represent the magnitude of the parameters, and $\phi_{1}, \phi_{2}, \phi_{3}, \phi_{4}, \phi_{5}, \phi_{6} \in[-\pi, \pi]$ are their phase values. Employed coaxial to waveguide transitions are slightly different and the proposed method deals with their particular differences providing an accurate set of $S$-parameters for each one.

From a device point of view, the connection of both standards to the coaxial to waveguide transition can be interpreted as a cascade of devices. The defined lines are considered lossless, which is a good approximation for aluminium and short waveguide sections used in this study.

The cascade formulas for $S$-parameters are well known and can be found in the literature [31].

\section{B. Optimization Techniques}

The flowchart of the optimization procedure employed in this study is shown in Fig. 2. The values of $S_{i j}^{\text {trn }}(n=1,2$ and $i, j=1,2$ ) are sequentially obtained for 1001 frequency points in the range under study with the help of two different optimization techniques. For the first frequency point, the initial solution is obtained with the aid of a genetic algorithms tool implemented in MATLAB [32]. After this stage, a more accurate refinement is obtained with the aid of a gradient descent optimization method by using as initial point for the search procedure the best 


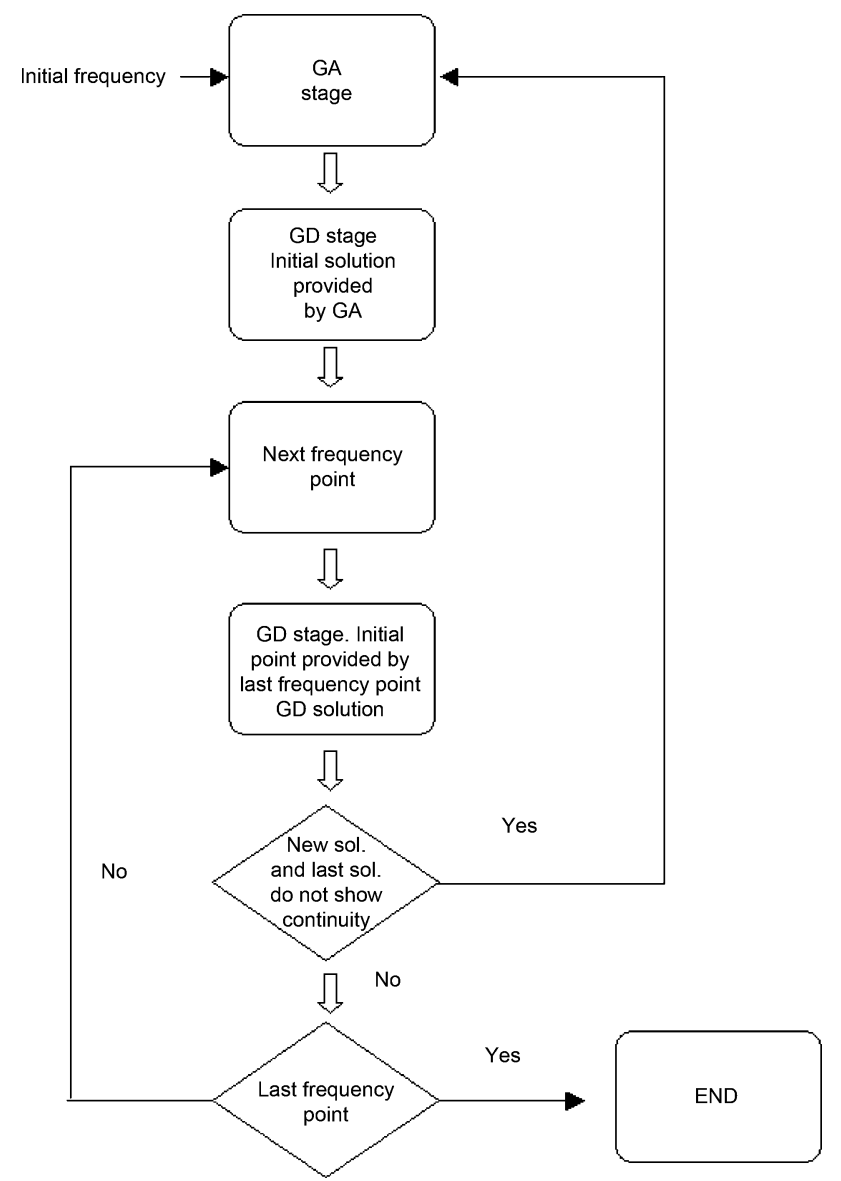

Fig. 2. Flowchart of the optimization procedure used in this study. GA: genetic algorithms. GD: gradient descent method.

solution obtained in the genetic algorithms' stage. Once the solution for the first frequency point is obtained with the required accuracy, the solution of the following frequency point is calculated with the gradient descent method by using as initial point the solution of the preceding frequency point iteratively. It is assumed then that the solution shows a slow variation; otherwise a sharp deviation may lead to wrong solution values increasing the fitness function evaluation. In this case, a genetic algorithms stage is generated again for the wrong last frequency point calculation.

Genetic algorithms are robust optimization tools based in the principles and concepts of natural selection. A global minimum can be reached in a solution space with weaker minima with the aid of these search procedures. In fact, a previous study for deembedding and unterminating symmetrical microwave fixtures using genetic algorithms can be found in [33], although the approach is different and only one thru standard is employed. Therefore, genetic algorithms have been chosen in this study in order to find the global minimum error at the first optimization stage.

An individual of genetic algorithms represents in this study a possible solution of our problem that contains the estimation of 12 different unknown real parameters necessary to characterize the two transitions. In a first stage, a random initial population with 100 individuals is evaluated. After this stage, crossover and mutation operations define the following generations evolving towards the optimum individual (our solution).

In our case, 12 unknown parameters require a high number of individuals and generations to reach a good approximation. Thus, 200 generations, 100 individuals, 80 crossovers per generation, and five mutations per generation have been used during the genetic algorithms' stage.

The gradient descent optimization stage uses a multivariable MATLAB function that implements the quasi-Newton method.

The evaluation function becomes a crucial aspect in both genetic algorithms and gradient descent optimization stages. In this study, the evaluation function takes into account the magnitude of the difference between the measured complex scattering parameters $\left(S_{i j}^{m}\right)$ and the simulated ones $\left(S_{i j}^{s}\right)$ for different experimental setups.

The expression used to evaluate the error when using the transition connected to a short-circuit standard during the optimization procedure is shown in (3). To evaluate a line connection between transitions, (4) is used. The evaluation function expression including all the error contributions is shown in (5). The evaluation is carried out for each frequency point

$$
\begin{aligned}
f_{\text {short }} & =\left|S_{11}^{m}-S_{11}^{s}\right|^{2} \\
f_{\text {thru/line }} & =\sum_{i=1}^{2} \sum_{j=1}^{2}\left|S_{i j}^{m}-S_{i j}^{s}\right|^{2} \\
f & =\sqrt{f_{\text {thru }}+f_{\text {line } 1}+f_{\text {line } 2}+f_{\text {short } 1}+f_{\text {short } 2}+f_{\text {short } 3}} .
\end{aligned}
$$

Different standard load combinations have been considered in order to assess the precision of this unterminating technique versus the number and type of used standards. Therefore, if any of the standards is not used during the optimization process, its contribution to function $f$ will be considered null.

\section{EXPERIMENTAL SETUP}

Fig. 3 shows the schemes of different experimental scenarios used to obtain the $S$-parameters of the transition and their identifications. Let $s 1$ be a short circuit placed on the waveguide port of both transitions, and $s 2$ and $s 3$ short circuits placed at 1.819 and $5.456 \mathrm{~cm}$ from that waveguide port, respectively. Additionally, let $t$ be the thru connection between the two coaxial to waveguide transitions, and $l 1$ and $l 2$ be $2-$ and $12.65-\mathrm{cm}-l e n g t h$ WR-340 waveguide lines, respectively.

A Rohde \& Schwarz ZVM VNA has been used in this study in order to measure the scattering matrix frequency behavior of the coaxial to waveguide transitions when using different standards. 1001 frequency points were collected in each measurement in the 2-3-GHz frequency range. Therefore, it was ensured that waveguide components worked only with the $\mathrm{TE}_{10}$ main mode.

A Rohde \& Schwarz ZV-Z32 PC 3.5 fixed matched calibration kit was employed in order to calibrate the VNA at port 1 of transition 1 and port 2 of transition 2 . The coaxial to waveguide transitions that were used to carry out the study belong to a Continental Microwave WCK340-HP waveguide calibration kit [34]. 


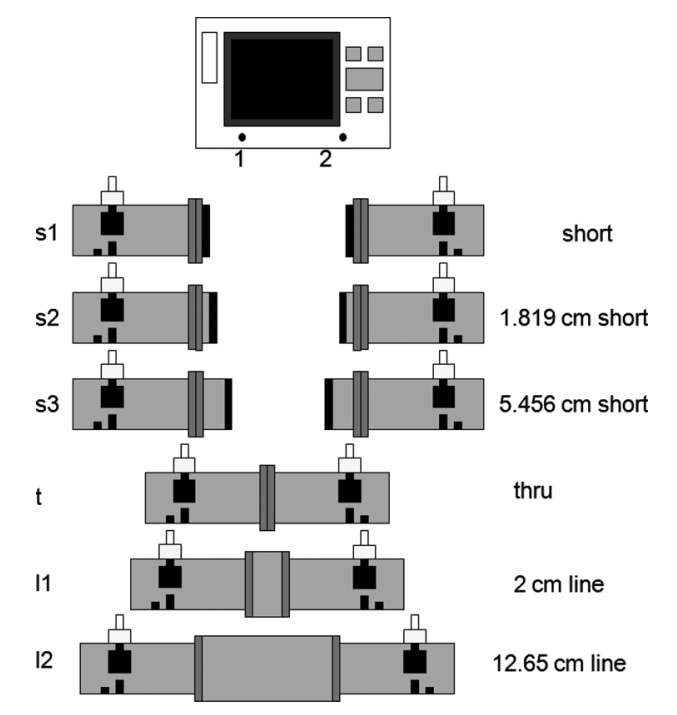

Fig. 3. Scheme of different calibration standards used for inverse measurements.

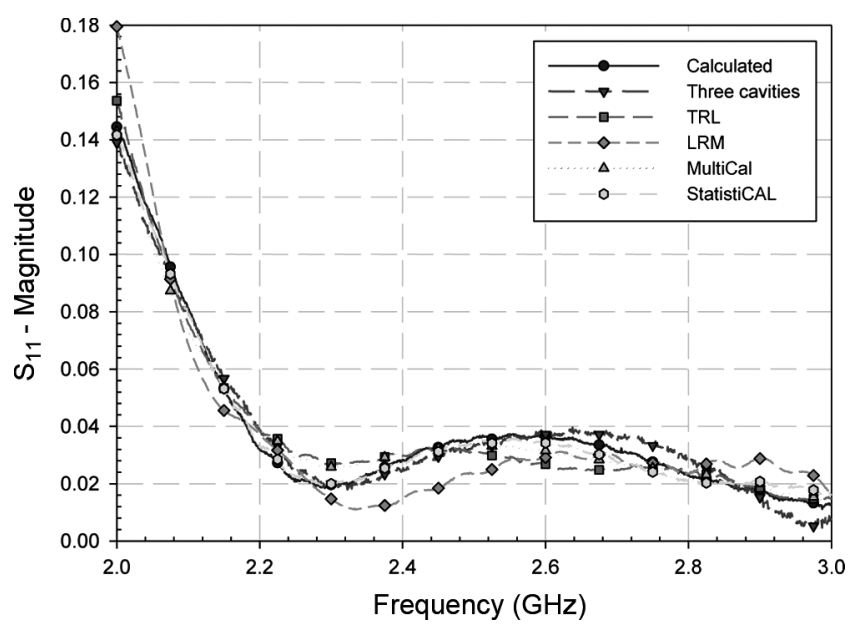

Fig. 4. $S_{11}$ results for transition 1 (magnitude).

The inverse procedure lasted from 2 to 14 min on an Intel Core Duo processor $2 \mathrm{GHz}$ depending on the set of the calibration standards employed.

\section{RESUltS AND DisCUSSION}

Figs. 4-7 show the results for transition 1 for both the magnitude and phase of the $S_{11}$ and $S_{12} S_{21}$ product when all short circuits and lines $(t, l 1, l 2, s 1, s 2, s 3)$ are used to evaluate the fitness function. The results of this new inverse technique are compared to those provided by the three cavities method [11], TRL [35], [36], line-reflect-match (LRM) [37] and MultiCal [38] and StatistiCAL [30] software packages. In the case of MultiCal and StatistiCAL, the number and type of standards are the same as those used in the proposed calibrating technique. From these results, it can be appreciated that all techniques show very good agreement. $S_{22}$ offers very similar results to $S_{11}$, and consequently, has not been depicted. The $S_{12} S_{21}$ product has been included in this study in order to be able to compare our results to the three cavities technique described in [11].

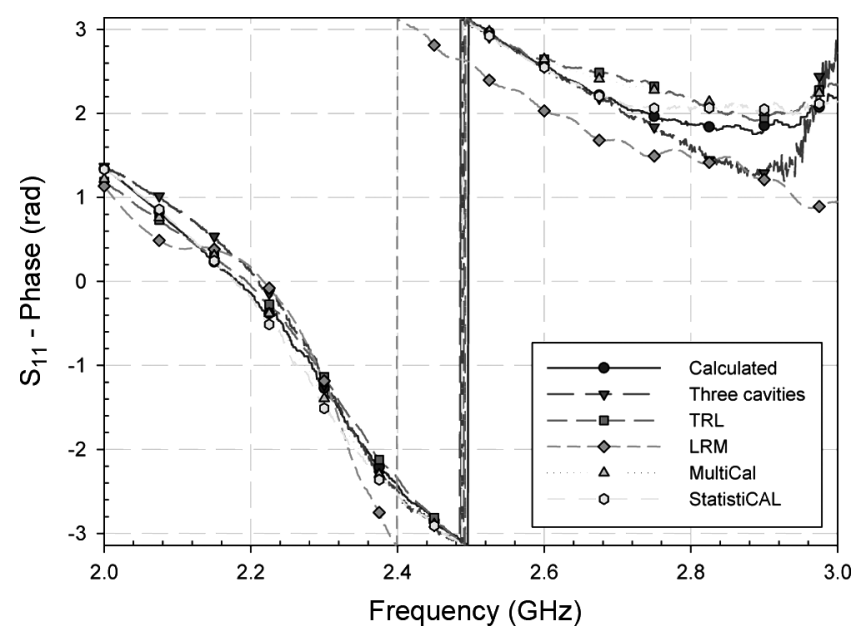

Fig. 5. $S_{11}$ results for transition 1 (phase).

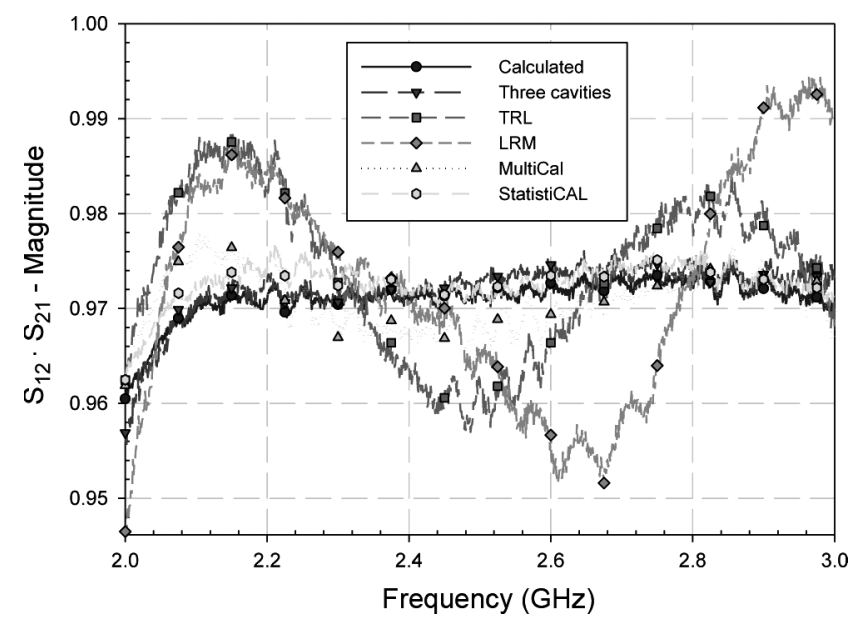

Fig. 6. $S_{12} \cdot S_{21}$ results for transition 1 (magnitude).

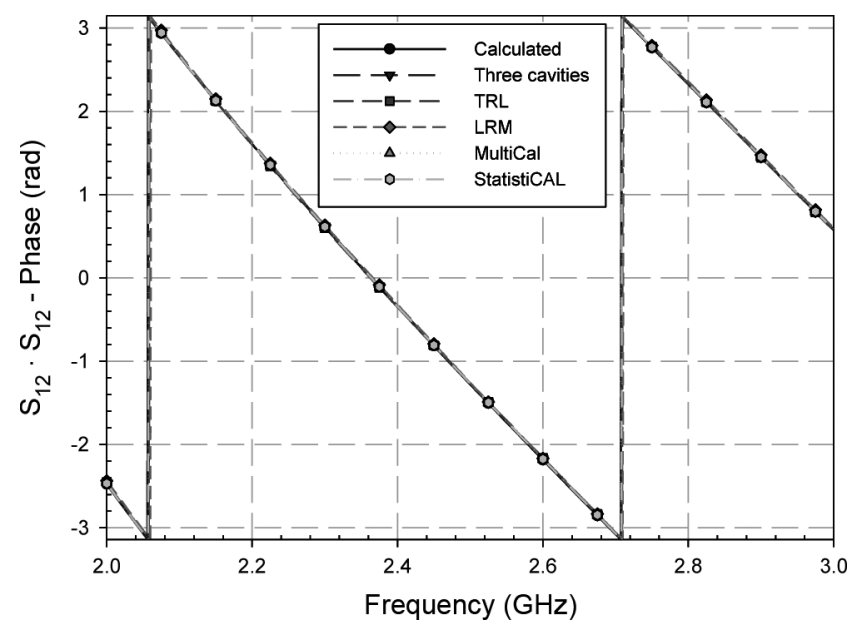

Fig. 7. $S_{12} \cdot S_{21}$ results for transition 1 (phase).

From Figs. 4 and 5, one can deduce that the main source of error is provided by $S_{11}$ phase uncertainties around $2.9 \mathrm{GHz}$ due to the high degree of matching of the transition at those frequencies. However, this is common to all the used techniques and can be explained as follows: if the noise level is near the 
TABLE I

Average AbSOlute ERror FOR DifFERENT ScENARIOS COMPARED TO THREE CAVITIES TECHNIQUE

\begin{tabular}{ccccc}
\hline \hline Scenarios/Error & $\left|\mathrm{S}_{I I}\right|$ & $\begin{array}{c}S_{11} \text { Phase } \\
(\mathrm{rad})\end{array}$ & $\left|\mathrm{S}_{12} \cdot \mathrm{S}_{2 I}\right|$ & $\begin{array}{c}S_{12} \cdot S_{21} \\
\text { Phase (rad) }\end{array}$ \\
\hline $\mathrm{t}, 11,12, \mathrm{~s} 1, \mathrm{~s} 2, \mathrm{~s} 3$ & 0.0036 & 0.1682 & 0.0012 & 0.0117 \\
$\mathrm{t}, 11, \mathrm{~s} 1, \mathrm{~s} 2, \mathrm{~s} 3$ & 0.0034 & 0.1501 & 0.0010 & 0.0036 \\
$\mathrm{t}, 11,12, \mathrm{~s} 1, \mathrm{~s} 2$ & 0.0046 & 0.1640 & 0.0023 & 0.0159 \\
$\mathrm{t}, 11, \mathrm{~s} 1, \mathrm{~s} 2$ & 0.0039 & 0.1440 & 0.0030 & 0.0061 \\
$\mathrm{t}, \mathrm{s} 1, \mathrm{~s} 2, \mathrm{~s} 3$ & 0.0025 & 0.1582 & 0.0019 & 0.0032 \\
$\mathrm{t}, 11,12, \mathrm{~s} 1$ & 0.0053 & 0.2293 & 0.0051 & 0.0165 \\
$\mathrm{t}, 11,12$ & 0.0053 & 0.2563 & 0.0069 & 0.1612 \\
$\mathrm{~s} 1, \mathrm{~s} 2, \mathrm{~s} 3$ & $1.9 \times 10^{-5}$ & 0.0028 & $1.8 \times 10^{-5}$ & $2.4 \times 10^{-5}$ \\
$\mathrm{t}, 11, \mathrm{~s} 1$ & 0.0053 & 0.2540 & 0.0066 & 0.0092 \\
$\mathrm{t}, \mathrm{s} 1, \mathrm{~s} 2$ & 0.0060 & 0.2509 & 0.0070 & 0.0089 \\
$\mathrm{t}, 11$ & 0.0058 & 0.2558 & 0.0075 & 0.1925 \\
$\mathrm{~s} 1, \mathrm{~s} 2$ & 0.1453 & 0.9644 & 0.1744 & 0.1771 \\
$\mathrm{t}, \mathrm{s} 1$ & 0.1983 & 0.3409 & 0.0360 & 6.1159 \\
$\mathrm{t}$ & 0.3281 & 0.7659 & 0.2404 & 0.2730 \\
$\mathrm{~s} 1$ & 0.3873 & 0.9957 & 0.3727 & 5.9391
\end{tabular}

reflected signal level, then the actual value of the imaginary and real parts of $S_{11}$ would be poorly estimated whatever the used technique.

Table I shows the frequency average absolute error of the proposed technique when comparing to the three cavities method described in [11] for different standards combinations used during inverse measurement. Both magnitude and phase errors are provided for $S_{11}$ and $S_{12} S_{21}$. Since there are many possible combinations, just some of them have been evaluated.

The inverse technique provides very low absolute error values for $S_{11}$ magnitude and $S_{12} S_{21}$ magnitude and phase when working with at least three different calibration standards. Again, in this case, the main error source is the $S_{11}$ phase due to its high level of matching.

Mixed combinations of shorts and thrus provide very similar results as long as there are enough conditions to obtain a valid solution.

As expected, the calibration with three shorts shows the minimum error values since the three cavities technique also uses three shorts as calibration standards [11]. However, very good results can be obtained even with different standards combinations, as shown in Figs. 4-7 and Table I.

When obtaining the transition $S$ parameters by using only three or two standards, there are some problems to follow the phase of the $S_{12} S_{21}$ function. Finally, from results obtained in Table I, it is obvious that if there is not enough information, the error increases. This can be observed in the last four cases of Table I. In those cases, the solution that minimizes the evaluation function cannot be reached by the proposed optimization algorithm.

Including intermediate restrictions in the cascading procedure for the transition parameters such as $\left|S_{i j}\right| \leq 1$ and $\left|S_{11}\right|^{2}+$ $\left|S_{12}\right|^{2} \leq 1$ vary neither the error values, nor convergence speed.

Table II has been obtained by comparing the solution obtained with the proposed technique to the solution obtained using a TRL calibration $(t, s 1, l 1)$. The comparison has been carried out for the same set of combinations of standards as in Table I. From the obtained results, it can be perceived that error values decrease when using $(t, s 1, l 1)$ with the proposed technique as the same standards as the TRL being employed to obtain the
TABLE II

AVERAge Absolute ERROR FOR DIFFERENT ScENARIOS COMPARED TO TRL CALIBRATION

\begin{tabular}{ccccc}
\hline \hline Scenarios/Error & $\left|\mathrm{S}_{11}\right|$ & $\begin{array}{c}S_{11} \text { Phase } \\
\text { (rad) }\end{array}$ & $\left|\mathrm{S}_{12} \cdot \mathrm{S}_{2 l}\right|$ & $\begin{array}{c}S_{12} \cdot S_{21} \\
\text { Phase (rad) }\end{array}$ \\
\hline t, 11, 12, s1, s2, s3 & 0.0044 & 0.1315 & 0.0076 & 0.0107 \\
t, 11, s1, s2, s3 & 0.0039 & 0.1346 & 0.0074 & 0.0102 \\
t, 11, 12, s1, s2 & 0.0041 & 0.1447 & 0.0068 & 0.0105 \\
t, 11,s1,s2 & 0.0030 & 0.1469 & 0.0062 & 0.0070 \\
t, s1, s2, s3 & 0.0055 & 0.1929 & 0.0081 & 0.0106 \\
t, 11, 12, s1 & 0.0048 & 0.1917 & 0.0029 & 0.0110 \\
t, 11, 12 & 0.0027 & 0.0808 & 0.0109 & 0.1615 \\
s1, s2, s3 & 0.0059 & 0.2563 & 0.0077 & 0.0107 \\
t, 11, s1 & 0.0032 & 0.1939 & 0.0014 & 0.0026 \\
t, s1, s2 & 0.0067 & 0.2856 & 0.0117 & 0.0127 \\
t, 11 & 0.0002 & 0.0105 & 0.0122 & 0.1904 \\
s1, s2 & 0.1434 & 0.7627 & 0.1797 & 0.1685 \\
t, s1 & 0.1989 & 0.4225 & 0.0427 & 6.1215 \\
t & 0.3287 & 0.8964 & 0.2412 & 0.2794 \\
s1 & 0.3829 & 0.7940 & 0.3758 & 5.9446
\end{tabular}

TABLE III

Average Absolute ERROR FOR DIFFERENT SCENARIOS COMPARED TO StatistiCAL SOFTWARE

\begin{tabular}{ccccc}
\hline \hline Scenarios/Error & $\left|\mathrm{S}_{I 1}\right|$ & $\begin{array}{c}S_{11} \text { Phase } \\
(\mathrm{rad})\end{array}$ & $\left|\mathrm{S}_{12} \cdot \mathrm{S}_{21}\right|$ & $\begin{array}{c}S_{12} \cdot S_{21} \\
\text { Phase (rad) }\end{array}$ \\
\hline t, 11, 12, s1, s2, s3 & 0.0020 & 0.0953 & 0.0016 & 0.0075 \\
t, 11, s1, s2, s3 & 0.0017 & 0.1301 & 0.0012 & 0.0027 \\
t, 11, 12, s1, s2 & 0.0022 & 0.1112 & 0.0021 & 0.0095 \\
t, 11,s1,s2 & 0.0025 & 0.1453 & 0.0018 & 0.0028 \\
t, s1, s2, s3 & 0.0022 & 0.1305 & 0.0014 & 0.0035 \\
t, 11, 12, s1 & 0.0031 & 0.2278 & 0.0020 & 0.0116 \\
t, 11, 12 & 0.0009 & 0.0305 & 20.9473 & 2.5445 \\
s1, s2, s3 & - & - & - & - \\
t, 11, s1 & 0.0033 & 0.1932 & 0.0017 & 0.0026 \\
t, s1, s2 & 0.0033 & 0.1645 & 0.0019 & 0.0071 \\
t, 11 & 0.0002 & 0.0089 & 1.0068 & 2.5687 \\
s1, s2 & - & - & - & - \\
t, s1 & - & - & - & - \\
t & - & - & - & - \\
s1 & - & - & - & -
\end{tabular}

solution. A similar behavior as shown in Table I can be observed for the rest of combinations.

Table III shows the comparison of the results provided by the proposed characterization method and those obtained by using the StatistiCAL software package for different standards' combinations. This software package was not able to provide results when there were not enough calibration standards, and consequently, some comparisons were not possible. It must be remarked that shorts $s 2$ and $s 3$ were introduced as loads since StatistiCAL only allows one reflection standard.

From obtained results, it can be observed that errors are very similar to those provided by the comparison to the previous methods. However, StatistiCAL is not able to provide results for some standards combinations, whereas the proposed method shows more flexibility in this field. Additionally for some standards (for instance, when using only lines of different lengths) StatistiCAL is not able to properly characterize $S_{12} S_{21}$.

Therefore, we have observed several advantages of the proposed method versus StatistiCAL. Firstly, this software needs a good initial value to provide accurate results, mainly for phase estimations. Secondly, the proposed method is able to handle more standards combinations without the need of an initial estimation for the transition scattering parameters. Additionally, the proposed method shows more flexibility in the error estimation since the evaluation function $(f)$ can be changed. StatistiCAL, 
TABLE IV

Average Absolute ERror for SCENARIO $t, l 1, l 2, s 1, s 2, s 3$ COMPARED TO DIFFERENT TECHNIQUES

\begin{tabular}{ccccc}
\hline \hline Technique/Error & $\left|\mathrm{S}_{I I}\right|$ & $\begin{array}{c}S_{11} \text { Phase } \\
(\mathrm{rad})\end{array}$ & $\left|\mathrm{S}_{12} \cdot \mathrm{S}_{2 I}\right|$ & $\begin{array}{c}S_{12} \cdot S_{21} \\
\text { Phase }(\mathrm{rad})\end{array}$ \\
\hline Three cavities & 0.0036 & 0.1682 & 0.0012 & 0.0117 \\
TRL & 0.0044 & 0.1315 & 0.0076 & 0.0107 \\
LRM & 0.0082 & 0.4590 & 0.0102 & 0.0269 \\
MultiCal & 0.0039 & 0.1230 & 0.0026 & 0.0039 \\
StatistiCAL & 0.0020 & 0.0953 & 0.0016 & 0.0075
\end{tabular}

however, shows lower computational times to provide the transition characterization. In fact, StatistiCAL showed computation times around $30 \mathrm{~s}$ in a 2-GHz Intel Core Duo processor.

Finally, Table IV provides a comparison between the three cavities method, TRL, LRM, MultiCal, and StatistiCAL. In this case, all the standards are employed for the proposed technique, MultiCal and StatistiCAL. A TRL and an LRM have been implemented with the calibration standards $(t, s 1, l 1)$ and $(t, s 1, m 1)$, respectively, with $m 1$ being the measurements for the termination provided by the calibration kit [34]. Differences for the phase value when using the LRM calibration can be observed in Fig. 5 for $S_{11}$ and may be due to the limitations of the employed match, as this adapted load behavior is not guaranteed for the entire frequency range under study [34]. The error is lower when comparing with the results provided by StatistiCAL and slightly increases when using MultiCal. This can be explained by the StatistiCAL robustness against noisy measurements [38]. As in the TRL technique, there is no redundant information, the error increases slightly with respect to the methods developed at NIST. Similar error results are obtained for the explicit LRM and three-cavity techniques.

\section{CONCLUSION}

A new two-tier inverse technique for characterizing coaxial to waveguide transitions based on the use of genetic algorithms and the gradient descent method has been described and compared to different well-known calibration techniques. Very good results can be obtained by using several calibration standards such as short circuits or waveguide lines.

Obtained results show that this inverse technique provides very similar results to those obtained by the reference algorithms, and additionally, is able to handle more calibration standards combinations provided that they can be properly included in $S$-parameter cascade calculations.

In this study, the studied frequency range has been restricted to the range provided by the manufacturer in which the coaxial to waveguide transitions are assumed to work properly. This also ensures waveguide monomode conditions. However, wider frequency ranges and higher frequency points could be readily handled by the proposed technique, although this would lead to higher computing times.

Additionally, the studied transitions showed nonresonant and well-matched behaviors. Resonant transitions are expected to produce sharper variations both in phase and magnitude values, and consequently, the proposed algorithm would very often use genetic algorithms to predict a reasonable initial point of the transition parameters at many frequency points. As a result, this would again lead to higher computing times than the ones obtained in this study.

Although applied to coaxial to waveguide transitions, this inverse technique can be extended to other transition types, and therefore, further research is envisaged in that direction.

\section{REFERENCES}

[1] M. L. Majewski, R. W. Rose, and J. R. Scott, "Modeling and characterization of microstrip-to-coaxial transitions," IEEE Trans. Microw. Theory Tech., vol. MTT-29, no. 8, pp. 799-805, Aug. 1981.

[2] J. R. Souza and E. C. Talboys, " $S$-parameter characterisation of coaxial to microstrip transition," Proc. Inst. Elect. Eng.-Microw., Opt., Antennas, vol. 129, no. 1, pp. 37-40, Feb. 1982.

[3] S. E. Gourley and A. G. Chapman, "Broadband characterisation of coaxial to microstrip transitions," in 12th Eur. Microw. Conf., Oct. 1982, pp. 622-627.

[4] C. Capsalis, C. P. Chronopoulous, and N. K. Uzunoglu, "A rigorous analysis of a coaxial to shielded microstrip line transition," IEEE Trans. Microw. Theory Tech., vol. 37, no. 7, pp. 1091-1098, Jul. 1989.

[5] J. Chramiec and J. K. Piotrowski, "Novel approach to the characterization of coaxial-to-microstrip transitions," in 27th Eur. Microw. Conf., Oct. 1997, vol. 2, pp. 697-702.

[6] J. Chramiec, B. Janiczak, J. Komisarczuk, J. K. Piotrowski, and W. Gwarek, "CAD models of connectors and transitions used in hybrid microwave integrated circuits," in 28th Eur. Microw. Conf., Oct. 1998, vol. 1, pp. 457-462.

[7] F. Dagang, Z. Dong, and S. Yuxuan, "Accurate analysis of the coax-tomicrostrip transition by using FDTD method," in Int. Comput. Electromagn.and Its Appl. Conf., 1999, pp. 218-221.

[8] S. A. Wartenberg and Q. H. Liu, "A coaxial-to-microstrip transition for multilayer substrates," IEEE Trans. Microw. Theory Tech., vol. 52, no. 2, pp. 584-588, Feb. 2004.

[9] B. K. O'Neil and J. L. Young, "Evaluation of coplanar waveguide-tomicrostrip transitions for precision $S$-parameter measurements," $M i$ crow. Opt. Technol. Lett., vol. 50, no. 10, pp. 2667-2671, 2008.

[10] R. Torres-Torres, G. Hernandez-Sosa, G. Romo, and A. Sanchez, "Characterization of electrical transitions using transmission line measurements," IEEE Trans. Adv. Packag., vol. 32, no. 1, pp. 45-52, Feb. 2009.

[11] J. F. Liang, H. Chang, and K. A. Zaki, "Coaxial probe modeling in waveguides and cavities," IEEE Trans. Microw. Theory Tech., vol. 40, no. 12, pp. 2172-2180, Dec. 1992.

[12] M. Hajian, D. P. Tran, and L. P. Ligthart, "Modeling the transition between a coaxial line and a flat rectangular waveguide," in 9th Int. Antennas Propag. Conf., Apr. 4-7, 1995, vol. 1, pp. 269-272.

[13] H. W. Yao and K. A. Zaki, "Modeling generalized coaxial probes in rectangular waveguides," in IEEE MTT-S Int. Microw. Symp. Dig., May 16-20, 1995, vol. 2, pp. 979-982.

[14] Y. F. Huang, H. David, S. Raymond, and P. Protap, "Modeling of cavity-backed coaxial line to rectangular waveguide junction," Int. J. RF Microw. Comput.-Aided Eng., vol. 8, no. 1, pp. 20-26, 1998.

[15] J. Randa, "Comparison of adapter characterization methods," IEEE Trans. Microw. Theory Tech., vol. 47, no. 12, pp. 2613-2620, Dec. 1999.

[16] J. P. Hoffmann, P. Leuchtman, A. Kretz, J. Rüfenacht, and R. Vahldieck, "Characterization of coaxial adapters for $S$-parameter measurements," in 38th Eur. Microw. Conf., Oct. 2008, pp. 313-316.

[17] R. F. Bauer and P. Penfield, "De-embedding and unterminating," IEEE Trans. Microw. Theory Tech., vol. MTT-22, pp. 282-288, Mar. 1974.

[18] D. Williams, "De-embedding and unterminating microwave fixtures with nonlinear least squares," IEEE Trans. Microw. Theory Tech., vol. 38, pp. 787-791, Jun. 1990.

[19] R. B. Marks, "A multiline method of analyzer calibration," IEEE Trans. Microw. Theory Tech., vol. 39, no. 7, pp. 1205-1215, Jul. 1991.

[20] R. B. Marks and D. F. Williams, "Characteristic impedance determination using propagation constant measurement," IEEE Microw. Guided Wave Lett., vol. 1, no. 6, pp. 141-143, Jun. 1991.

[21] R. B. Marks and D. F. Williams, "Accurate transmission line characterization," IEEE Microw. Guided Wave Lett., vol. 3, no. 8, pp. 247-249, Aug. 1993.

[22] R. B. Marks and D. F. Williams, "Accurate experimental characterization of interconnects," IEEE Trans. Compon., Hybrids, Manuf. Technol., vol. 15, no. 4, pp. 601-602, Aug. 1992. 
[23] X. P. Liang, K. A. Zaki, and A. E. Atia, "A rigorous three plane modematching technique for characterizing waveguide T-junctions, and its application in multiplexer design," IEEE Trans. Microw. Theory Tech., vol. 39, no. 12, pp. 2138-2147, Dec. 1991.

[24] A. A. Kirilenko, S. L. Senkevich, V. I. Tkachenko, and B. G. Tysik, "Waveguide diplexer and multiplexer design," IEEE Trans. Microw. Theory Tech., vol. 42, no. 7, pp. 1393-1396, Jul. 1994.

[25] F. Arndt, I. Ahrens, U. Papziner, U. Wiechmann, and R. Wilkeit, "Optimized E-plane T-junction series power dividers," IEEE Trans. Microw. Theory Tech., vol. MTT-35, no. 11, pp. 1052-1059, Nov. 1987.

[26] G. Gerini and M. Guglielmi, "Full-wave CAD of a rectangular waveguide filter with integrated coaxial excitation," IEEE Trans. Microw. Theory Tech., vol. 49, no. 5, pp. 986-989, May 2001

[27] G. Gerini, F. D. Bustamante, and M. Guglielmi, "Triple mode filters with coaxial excitation," in IEEE MTT-S Int. Microw. Symp. Dig., 2000, vol. 3, pp. 1763-1766.

[28] Z. Shen and C. Feng, "A new dual-polarized broadband horn antenna," IEEE Antennas Wireless Propag. Lett., vol. 4, pp. 270-273, 2005.

[29] M. E. Requena-Pérez, A. Albero-Ortiz, J. Monzó-Cabrera, and A. Díaz-Morcillo, "Combined use of genetic algorithms and gradient descent optimization methods for accurate inverse permittivity measurement," IEEE Trans. Microw. Theory Tech., vol. 54, no. 2, pp. 615-624, Feb. 2006.

[30] D. F. Williams, J. C. M. Wang, and U. Arz, "An optimal vector-network-analyzer calibration algorithm," IEEE Trans. Microw. Theory Tech., vol. 51, no. 12, pp. 2391-2401, Dec. 2003.

[31] K. Rothemund, H. W. Glock, and U. van Rienen, "Eigenmode calculation of complex RF-structures using $S$-parameters," IEEE Trans. Magn., vol. 36, no. 4, pp. 1501-1503, Jul. 2000.

[32] C. R. Houck, J. A. Joines, and M. G. Kay, "A genetic algorithm for function optimization: A MATLAB implementation," The Mathworks, Natick, MA, NCSU-IE TR 95-09, 1995.

[33] A. S. Adalev, N. V. Korovkin, M. Hayakawa, and J. B. Nitsch, "Deembedding and unterminating microwave fixtures with the genetic algorithm," IEEE Trans. Microw. Theory Tech., vol. 54, no. 7, pp. 3131-3140, Jul. 2006.

[34] Waveguide Component Specifications and Design Handbook, \#7 ed. Dorset, U.K.: Cobham, 2009, Continental Microw.

[35] G. F. Engen and C. A. Hoer, "Thru-reflect-line: An improved technique for calibrating the dual six-port automatic network analyzer," IEEE Trans. Microw. Theory Tech., vol. MTT-27, no. 12, pp. 987-993, Dec. 1979.

[36] M. L. Edwards, "Calibration and measurements of $S$-parameters," in Microwave \& RF Circuits: Analysis, Design, Fabrication \& Measurement. Baltimore, MD: The Johns Hopkins Univ. Press, 2001, ch. 7.

[37] K. Silvonen, "LMR 16-A self calibration procedure for a leaky network analyzer," IEEE Trans. Microw. Theory Tech., vol. 45, no. 7, pp. 1041-1049, Jul. 1997.

[38] D. F. Williams, C. M. Wang, and U. Arz, "An optimal multiline TRL calibration algorithm," in IEEE MTT-S Int. Microw. Simp. Dig., Jun. 2003, vol. 3, pp. 1819-1822.

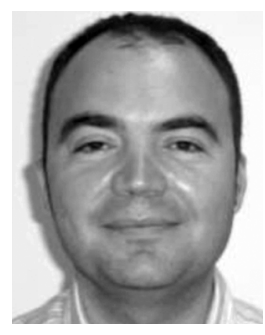

Antonio José Lozano-Guerrero was born in El Verger, Spain, in 1976. He received the Dipl. Ing. degree in telecommunications engineering from the Universidad Politécnica de Valencia (UPV), Valencia, Spain in 2003 and the Ph.D. degree from the Universidad Politécnica de Cartagena (UPCT), Cartagena, Spain in 2008

From 2003 to 2004, he was a Research Assistant with the Department of Communications, UPV. In 2004, he joined the Departamento de Tecnologías de la Información y las Comunicaciones, UPCT, where he is currently an Associate Lecturer. His current research areas are electromagnetic compatibility, numerical techniques in electromagnetism, and industrial microwave heating systems.

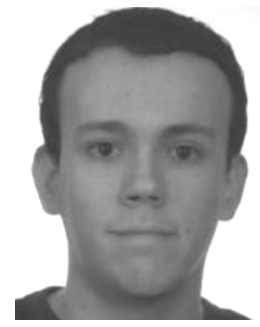

Francisco Javier Clemente-Fernández was born in La Unión (Murcia), Spain, in 1985. He received the Dipl. Ing. degree in telecommunications engineering from the Universidad Politécnica de Cartagena (UPCT), Cartagena, Spain, in 2008, and is currently working toward the Ph.D. degree at UPCT.

In 2008, he joined the Departamento de Tecnologías de la Información y las Comunicaciones, UPCT. His main research areas are microwave-assisted heating and drying processes and microwave filters design and optimization.

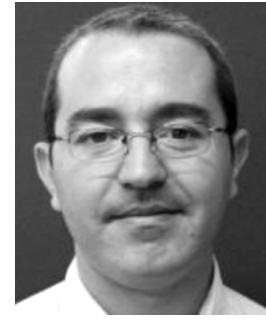

Juan Monzó-Cabrera (M'09) was born in Elda (Alicante), Spain, in January 1973. He received the Dipl. Ing. and Ph.D. degrees in telecommunications engineering from the Universidad Politécnica de Valencia, Valencia, Spain.

$\mathrm{He}$ is currently an Associate Lecturer with the Departamento de Tecnologías de la Información y las Comunicaciones, Universidad Politécnica de Cartagena, Cartagena, Spain. He is a Director of the Association of Microwave Power in Europe for Research and Education (AMPERE), a European association devoted to RF and microwave energy promotion. He has coauthored over 60 papers in referred journals and conference proceedings. He holds several patents regarding microwave heating processes. His current research areas cover microwave-assisted heating and drying processes and numerical techniques in electromagnetism.

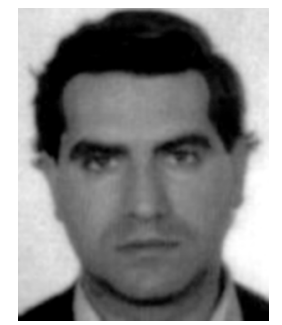

Juan Luis Pedreño-Molina was born in Cartagena (Murcia), Spain, in 1969. He received the BASc. degree and $\mathrm{Ph} . \mathrm{D}$. degree in neurotechnology, control, and robotics from the Technical University of Madrid (UPM), Madrid, Spain, in 1984 and 2000, respectively.

He currently an Assistant Professor of telecommunication engineering with the Universidad Politécnica de Cartagena (UPCT), Cartagena, Spain. Since 1999, he has been with the Departamento de Tecnologías de la Información y las Comunicaciones, UPCT. His research interests are signal processing applied to optimization and control of nonlinear systems and modeling based on neural networks with applications to robotics and drying processes.

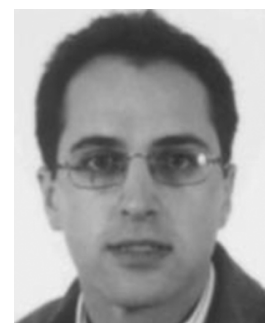

Alejandro Díaz-Morcillo (S'95-M'02-SM'09) was born in Albacete, Spain, in 1971. He received the Ingeniero (Ms. Eng.) and Doctor Ingeniero (Ph. D.) degrees in telecommunication engineering from the Polytechnic University of Valencia (UPV), Valencia, Spain, in 1995 and 2000, respectively.

From 1996 to 1999, he was a Research Assistant with the Department of Communications, UPV. In 1999, he joined the Departamento de Tecnologías de la Información y las Comunicaciones, Universidad Politécnica de Cartagena (UPCT), Cartagena, Spain, as a Teaching Assistant. Since 2001, he has been an Associate Professor with UPCT, where he leads the Electromagnetics and Matter Research Group. His main research interests concern numerical methods in electromagnetics and industrial microwave heating systems. 